\title{
Integrin a6-Targeted Molecular Imaging of Central Nervous System Leukemia in Mice
}

\author{
Wenbiao Zhang ${ }^{1,2 \dagger}$, Yongjiang $\mathrm{Li}^{2,3 \dagger}$, Guanjun $\mathrm{Chen}^{2,4 \dagger}$, Xiaochun $\mathrm{Yang}^{3}$, Junfeng $\mathrm{Hu}^{3}$, \\ Xiaofei Zhang ${ }^{2,3 *}$, Guokai Feng ${ }^{2 *}$ and Hua Wang ${ }^{4 *}$
}

OPEN ACCESS

Edited by:

Gianni Ciofani,

Italian Institute of Technology, Italy

Reviewed by:

Wen Shi,

University of Nebraska Medical

Center, United States

Jianguo Lin,

Jiangsu Institute of Nuclear Medicine,

China

Yu Chunjing,

Affiliated Hospital of Jiangnan

University, China

*Correspondence:

Xiaofei Zhang

zhangxf1@sysucc.org.cn

Guokai Feng

fengguok@sysucc.org.cn

Hua Wang

wanghua@sysucc.org.cn

${ }^{+}$These authors have contributed equally to this work

Specialty section: This article was submitted to

Nanobiotechnology,

a section of the journal Frontiers in Bioengineering and

Biotechnology

Received: 10 November 2021

Accepted: 17 January 2022

Published: 23 February 2022

Citation:

Zhang W, Li Y, Chen G, Yang X, Hu J,

Zhang $X$, Feng $G$ and Wang $H$ (2022)

Integrin $\alpha 6$-Targeted Molecular

Imaging of Central Nervous System

Leukemia in Mice.

Front. Bioeng. Biotechnol. 10:812277.

doi: 10.3389/fbioe.2022.812277
${ }^{1}$ Department of Medical Imaging, Sun Yat-sen University Cancer Center, Guangzhou, China, ${ }^{2}$ State Key Laboratory of Oncology in South China, Collaborative Innovation Center for Cancer Medicine, Sun Yat-sen University Cancer Center, Guangzhou, China, ${ }^{3}$ Department of Nuclear Medicine, Sun Yat-sen University Cancer Center, Guangzhou, China, ${ }^{4}$ Department of Hematological Oncology, Sun Yat-sen University Cancer Center, Guangzhou, China

Central nervous system leukemia (CNS-L) is caused by leukemic cells infiltrating into the meninges or brain parenchyma and remains the main reason for disease relapse. Currently, it is hard to detect CNS-L accurately by clinically available imaging models due to the relatively low amount of tumor cells, confined blood supply, and the inferior glucose metabolism intensity. Recently, integrin a6-laminin interactions have been identified to mediate CNS-L, which suggests that integrin a6 may be a promising molecular imaging target for the detection of CNS-L. The acute lymphoblastic leukemia (ALL) cell line NALM6 stabled and transfected with luciferase was used to establish the CNS-L mouse model. CNS-L-bearing mice were monitored and confirmed by bioluminescence imaging. Three of our previously developed integrin a6-targeted peptide-based molecular imaging agents, Cy5-S5 for near-infrared fluorescence (NIRF), Gd-S5 for magnetic resonance (MR), and ${ }^{18} \mathrm{~F}-\mathrm{S} 5$ for positron emission tomography (PET) imaging, were employed for the molecular imaging of these CNS-L-bearing mice. Bioluminescence imaging showed a local intensive signal in the heads among CNS-Lbearing mice; meanwhile, Cy5-S5/NIRF imaging produced intensive fluorescence intensity in the same head regions. Moreover, Gd-S5/MR imaging generated superior MR signal enhancement at the site of meninges, which were located between the skull bone and brain parenchyma. Comparatively, MR imaging with the clinically available MR enhancer Gd-DTPA did not produce the distinguishable MR signal in the same head regions. Additionally, ${ }^{18} \mathrm{~F}$-S5/PET imaging also generated focal radio-concentration at the same head regions, which generated nearly 5 -times tumor-to-background ratio compared to the clinically available PET radiotracer ${ }^{18} \mathrm{~F}-\mathrm{FDG}$. Finally, pathological examination identified layer-displayed leukemic cells in the superficial part of the brain parenchyma tissue, and immunohistochemical staining confirmed the overexpression of the integrin $a 6$ within the lesion. These findings suggest the potential application of these integrin a6-targeted molecular imaging agents for the accurate detection of CNS-L.

Keywords: leukemia, molecular imaging, integrin $a 6$, positron emission tomography, central nervous system leukemia 


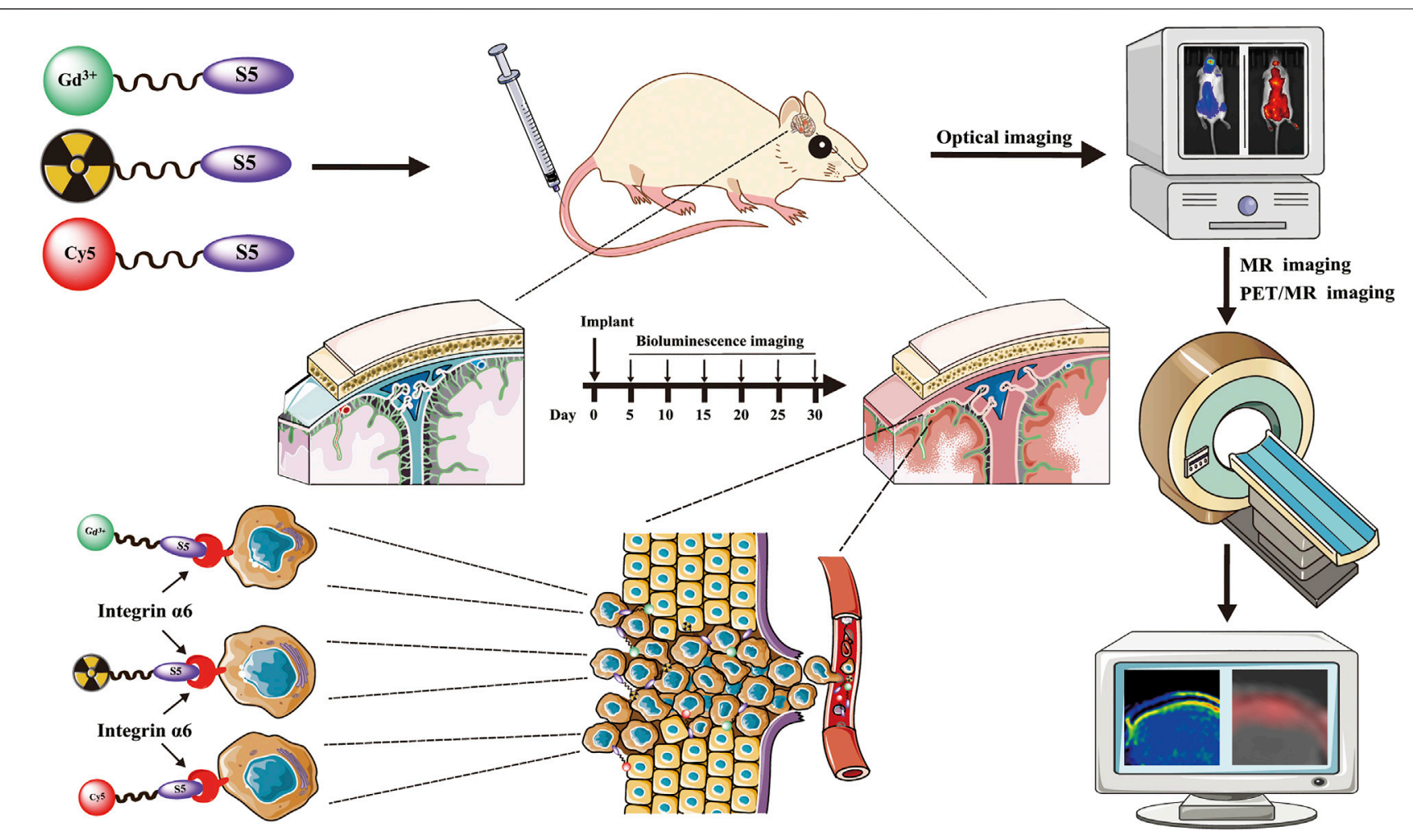

GRAPHICAL ABSTRACT

\section{INTRODUCTION}

Acute lymphoblastic leukemia (ALL) is a malignant proliferation of lymphocytes that could invade the bone marrow, blood, and extramedullary tissues or organs, with an estimated incidence of about $157 / 100,000$ and a distinctive prevalence in children, especially in those at 1-4 years old (Siegel et al., 2019; Malard and Mohty, 2020). About $80-90 \%$ patients could achieve a complete response after multiagent chemotherapy. However, central nervous system involvement of ALL, caused by leukemic cells infiltrating into the meninges or brain parenchyma, which is also called central nervous system leukemia (CNS-L), remains the main reason for disease relapse during the remission period. Because most chemotherapeutic drugs could not penetrate the blood-brain barrier, leukemic cells hidden in the central nervous system could not be effectively eliminated and become the origin of extramedullary leukemia recurrence (Pinnix et al., 2018; Zhang C et al., 2020). Nevertheless, accurate diagnosis of the central nervous system (CNS) involvement is a major clinical challenge that commonly leads to delayed or excessive treatment (Frishman-Levy and Izraeli, 2017; Jin et al., 2018). Due to the diagnosis insufficiency, prophylactic intrathecal chemotherapy (methotrexate in most regimens) is routinely administered for all patients in the clinical setting regardless of their CNS status, and chemotherapy-induced CNS toxicity always accompanied numerous adverse clinical manifestations including impaired consciousness, focal deficits, seizures, and headaches (Vagace et al., 2012). In addition, patients evaluated at a high risk of CNS infiltration routinely received additional cranial irradiation, which could further exacerbate neurocognitive impairment and the risk of secondary malignancies (Zhou et al., 2020).

The current gold standard for assessing CNS-L is pathological detection of leukemic cells in the cerebrospinal fluid through lumbar puncture (Bürger et al., 2003). However, the examination method has a relatively high false-negative rate, and the positivity is hysteretic than the primary CNS involvement, which highlights the urgent need for novel diagnostic methods. New strategies aimed at improving the diagnostic sensitivity of cerebrospinal fluid examination by qPCR or flow cytometry encountered methodological challenges (Yousafzai et al., 2019; Thastrup et al., 2020). Imaging diagnosis by detecting CNS lesions could be more district and intuitive; however, conventional imaging examinations including enhanced computerized tomography (CT), magnetic resonance imaging (MRI), or ${ }^{18} \mathrm{~F}-\mathrm{FDG}$ positron emission tomography (PET) showed limited ability in detecting CNS-L lesions because of a relatively low amount of tumor cells, confined blood supply, and inferior glucose metabolism intensity compared with other solid tumor lesions (Ranta et al., 2017a; Ranta et al., 2017b). Based on the premises, the development of a specific molecular probe for imaging could provide a novel way for the targeted imaging of CNS-L. 
Integrins are heterodimeric transmembrane receptors consisting of $\alpha$ and $\beta$ subunits, each with a single-pass transmembrane domain and a typically short cytoplasmic domain (Erdreich-Epstein et al., 2005; Zhu et al., 2007). Integrin $a 6$ is encoded by the ITGA6 gene and dimerizes with integrin $\beta 1$ or $\beta 4$ to form integrin $\alpha 6 \beta 1$ and $\alpha 6 \beta 4$ (Takada et al., $2007)$. Integrin $\alpha 6$ normally expresses on the cell surface and acts as the mediator of intracellular and extracellular matrix adhesion (Krebsbach and Villa-Diaz, 2017). Numerous studies have revealed that integrin $a 6$ was highly expressed in tumor cells of ALL patients and the corresponding cell lines (Blase et al., 1996). According to the GEPIA database, ITGA6 was significantly overexpressed in leukemia cells than the paired normal blood cells and the brain tissues (http://gepia.cancerpku.cn/). Recent studies have found that ALL chemoresistance was dependent on ALL cells adhering to the stroma through the adhesion of integrin $a 6$, and its expression mediated the invasion to the CNS tissues via neuromigration pathways (Yao et al., 2018; Gang et al., 2020; Lenk et al., 2020). Thus, integrin a6 could serve as a potential molecular target for the imaging of CNS-L lesions.

In our previous study, we have identified a peptide CRWYDENAC (dubbed RWY) that could bind specifically to integrin $\alpha 6$ by phage display technology (Feng $G$ et al., 2019). We further translated the RWY peptide into an integrin a6-targeted PET probe and MRI probe that have been successfully applied for tumor imaging of the integrin a6 expression (Feng GK et al., 2019; Xiao et al., 2019; Zhang Y et al., 2020; Gao et al., 2020). Recently, our group improved the characteristics of the peptide by alanine scanning and obtained a novel integrin a6-targeting peptide CRWYDANAC (dubbed S5) which exhibited a higher specificity and affinity to integrin a6 (Mei et al., 2020; Lin et al., 2021). On these bases, we further developed the targeted molecular imaging probes for near-infrared fluorescence (NIRF) imaging, MRI, and PET based on the S5 peptide, which is called Cy5-S5, Gd-S5, and ${ }^{18} \mathrm{~F}-\mathrm{S} 5$ in short. In this study, the affinity of the synthesized S5 peptide to ALL cell lines was verified, and the imaging of CNS-L mouse models using NIRF, MRI, and PET scanners was conducted to test the imaging efficacy of the targeted imaging probes for CNS-L.

\section{MATERIALS AND METHODS}

\section{Cells and Animals}

The human ALL cell lines including NALM6, Reh, Jurkat, and CCRF-CEM were purchased from the American Type Culture Collection (ATCC) and cultured in RPMI 1640 medium supplemented with $10 \%$ fetal bovine serum and $0.5 \%$ penicillin-streptomycin and incubated at $37{ }^{\circ} \mathrm{C} / 5 \% \mathrm{CO}_{2}$. To facilitate tumor monitoring, luminescent cells NALM6luciferase were generated by stable transfection with luciferase. Female NOD/SCID mice (6 weeks old) were purchased from Vita River, Charles River Laboratories, China (Beijing, China). NOD/ SCID mice were injected via the tail vein with $1 \times 10^{6}$ luciferased cells suspended in $150 \mu \mathrm{l}$ of sterile phosphate-buffered saline (PBS). Bioluminescence imaging was applied to monitor the leukemic burden. All animal experiments were approved by the Institutional Animal Care and Use Committee (IACUC) at the Sun Yat-sen University Cancer Center (IACUC approval number L102012020070M), and the welfare and treatment of the laboratory animals were in accordance with the corresponding animal management regulations.

\section{Preparation of S5 Peptide-Based Imaging Probes for NIRF, MR, and PET}

The integrin a6-targeted peptide S5 was synthesized by the standard Fmoc-based solid-phase synthesis and provided by Chinese Peptide Company (Hangzhou, China). In order to make a better combination of S5 and Gd and to reduce the complexity of the process, we used the $\mathrm{S} 5$ peptide sequence as a straight peptide in the synthesis of the peptide Gd-S5, while the other two synthetic peptides were cyclic peptides. Briefly, DOTAS5 or NOTA-S5 was synthesized through a condensation reaction between carboxyl groups in DOTA or NOTA and amine groups in reverse S5. The crude product was eluted with a gradient of acetonitrile, and the end product was purified by highperformance liquid chromatography (HPLC), followed by characterization using matrix-assisted laser desorption/ ionization time-of-flight (MALDI-TOF) mass spectrometry (Bruker Daltonics, Germany). Then, Cy5-S5 was compounded by conjugating the Cy5 to the $\mathrm{S} 5$ peptide through an amidation reaction, and Gd-S5 was synthesized by complexation of $\mathrm{Gd}$ with the DOTA-S5.

As ${ }^{18} \mathrm{~F}$ has a relatively fast rate of decay, ${ }^{18} \mathrm{~F}-\mathrm{S} 5$ would be synthesized each time before use by radiolabeling the NOTAS5 with ${ }^{18} \mathrm{~F}$. In brief, the S5 peptide solution was freeze-dried with the lyophilized kits, with an $8 \mathrm{nmol} \mathrm{S} 5$ peptide and $6 \mathrm{nmol}$ $\mathrm{AlCI}_{3} .6 \mathrm{H} 2 \mathrm{O}$ in each vial unit. The reaction procedure was performed as follows: 1 vial unit was added with $65 \mu$ of ${ }^{18} \mathrm{~F}$ (approximately $10 \mathrm{mCi}$ ) in DI water, $5 \mu$ of acetic acid, and $330 \mu \mathrm{l}$ ethanol and heated for $10 \mathrm{~min}$ at $100^{\circ} \mathrm{C}$. The reaction mixture was diluted with $10 \mathrm{ml}$ of water and trapped in a Varian Bond Elut C18 column (100 mg). The column was washed with $10 \mathrm{ml}$ of water and then eluted with $400 \mu \mathrm{l}$ of ethanol twice. Then, the eluate was air-dried by nitrogen. Finally, the end product was dissolved with PBS before injection.

\section{Flow Cytometry}

NALM6, Ref, Jurkat, and CCRF-CEM cells were seeded into six-well plates, respectively, and then incubated with $10 \mu \mathrm{l} /$ $10^{6}$ cell concentration of the anti-integrin a 6 antibody $(\mathrm{R} \& \mathrm{D}$, $\mathrm{FAB} 13501 \mathrm{P})$ and IgG2A (R\&D, IC006P) at $4^{\circ} \mathrm{C}$ for $30 \mathrm{~min}$, protected from light. After incubating for $30 \mathrm{~min}$, the cells were washed with PBS three times and resuspended in $500 \mu \mathrm{l}$ of PBS. The fluorescence intensity was measured by flow cytometry (Beckman Coulter, CytoFLEX S, United States).

\section{Immunofluorescence}

Approximately $1 \times 10^{6}$ NALM6 cells were seeded on cover slips in 24-well plates and incubated for $24 \mathrm{~h}$. Subsequently, the $80 \mu \mathrm{M}$ S5 peptide was added into the culture medium and incubated with cells for $4 \mathrm{~h}$, while the blank control group was 
A

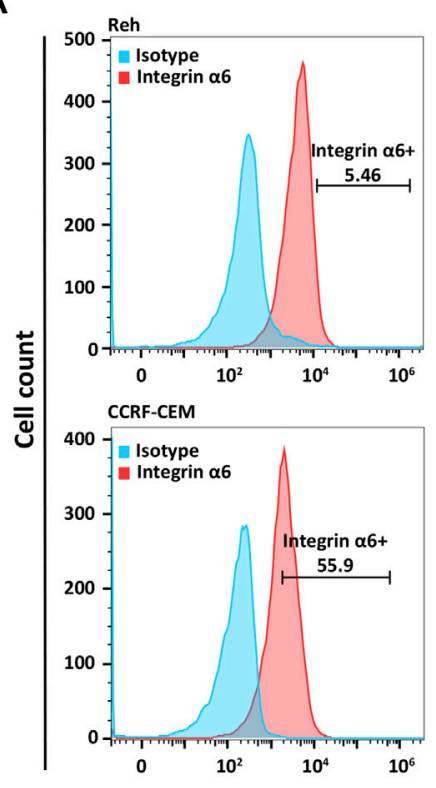

B
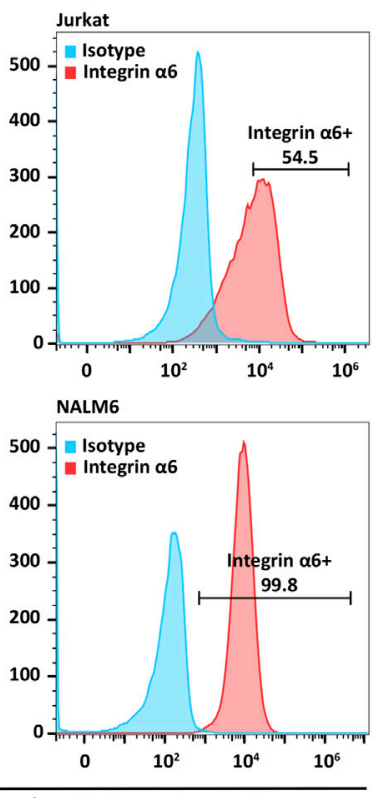

Anti-Integrin $\alpha 6-\mathrm{Pe}$

C

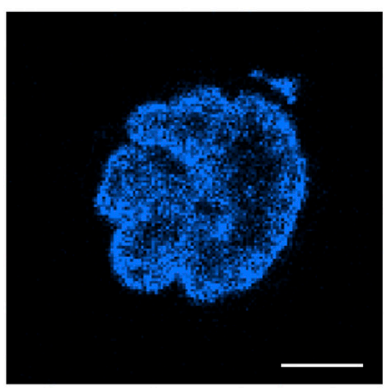

DAPI

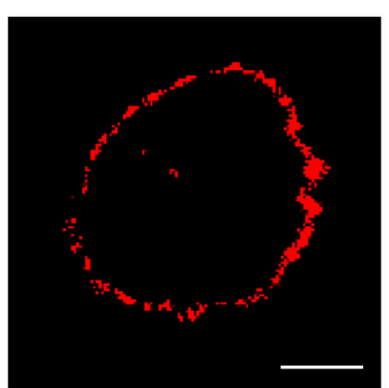

Integrin $\alpha 6$

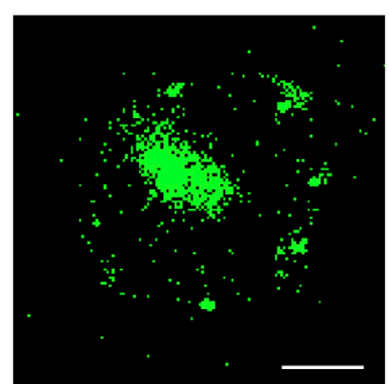

S5

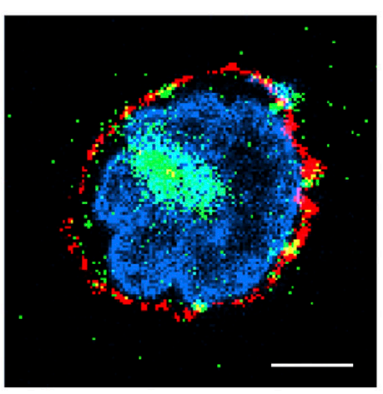

Merge

FIGURE 1 | Surface expression of integrin a6 in human ALL cell lines and binding of the S5 peptide to NALM6 cells. (A) Flow cytometry identified the surface expression of integrin a 6 in human ALL cell lines including NALM6, Ref, Jurkat, and CCRF-CEM, and (B) the highest expression of integrin a6 was observed in NALM6 cells. (C) Confocal microscopy confirmed the cellular localization of integrin a6 and the S5 peptide in NALM6 cells (Scale bar, $4 \mu \mathrm{m}$ ).

added to the same volume of RPMI 1640 medium. After that, cells of each group were washed with PBS five times, fixed with $4 \%$ paraformaldehyde for $15 \mathrm{~min}$, permeabilized with $0.1 \%$ Triton X-100 (Sigma-Aldrich, Germany), and blocked with $1 \%$ BSA for $30 \mathrm{~min}$. The cover slips were incubated with the anti-integrin a6 antibody (Abcam, ab181551) at $4^{\circ} \mathrm{C}$ overnight, followed by incubation with streptavidin-Cy3 (Thermo Fisher 434315, United States) and the goat antimouse Alexa Fluor 488 secondary antibody (Abcam, ab150113) for $1 \mathrm{~h}$ at room temperature in a dark chamber. Finally, cover slips were incubated with $1 \mu \mathrm{g} / \mathrm{ml}$ DAPI and mounted with slides with ProLong gold antifade (Invitrogen P26930, United States). Fluorescence images were visualized and captured under a confocal microscopy confocal laserscanning system (Zeiss, LSM980, Germany) at $40 \times$ and $100 \times$ magnification. Co-localization was analyzed by ImageJ (http://rsbweb.nih.gov/ij/) and the co-localization finder plug-in.

\section{NIRF Imaging}

Both bioluminescence and NIRF imaging are performed using IVIS Spectrum equipment manufactured by PerkinElmer, United States. We performed bioluminescence imaging on days $5,10,15,20,25$, and 30 after the tail vein injection of leukemic cells until the imaging results confirmed the presence of focal luminescence intensity around the head of the mice. Bioluminescence imaging was performed starting $5 \mathrm{~min}$ after the intraperitoneal injection of luciferin. NIRF was acquired with an excitation at $640 \mathrm{~nm}$ and emission at $680 \mathrm{~nm}$ (exposure time: $3 \mathrm{~s}$ ). Mice with CNS-L confirmed by bioluminescence were used for NIRF imaging studies on the following day. Mice were anesthetized with 3\% isoflurane and maintained under anesthesia with 1-2\% isoflurane and oxygen. An injection of Cy5-S5 and Cy5-CG7C solutions was freshly prepared in saline, and the solution was stabilized for $20 \mathrm{~min}$ before injection. Each mouse was injected intravenously with an equivalent of $10 \mathrm{nmol}(0.5 \mathrm{mg} / \mathrm{Kg})$ of Cy5-S5 and Cy5-CG7C. 


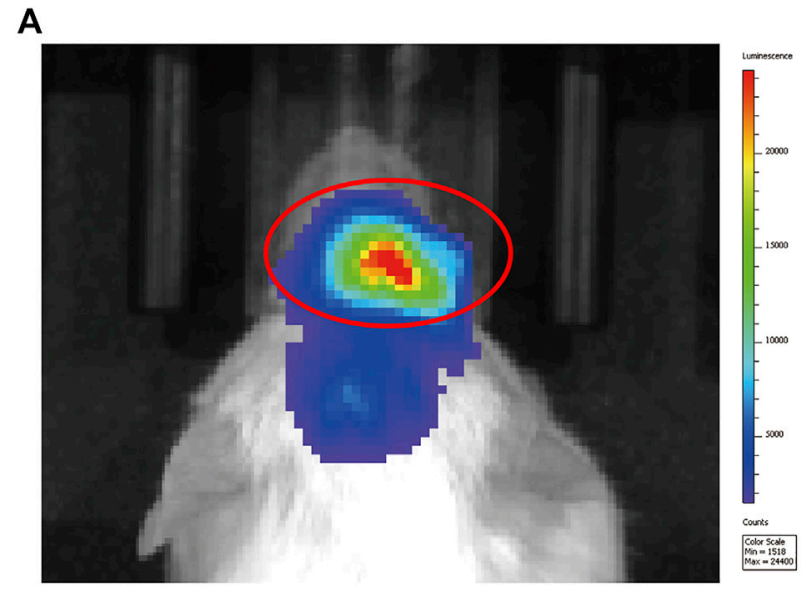

D-Luciferin

B

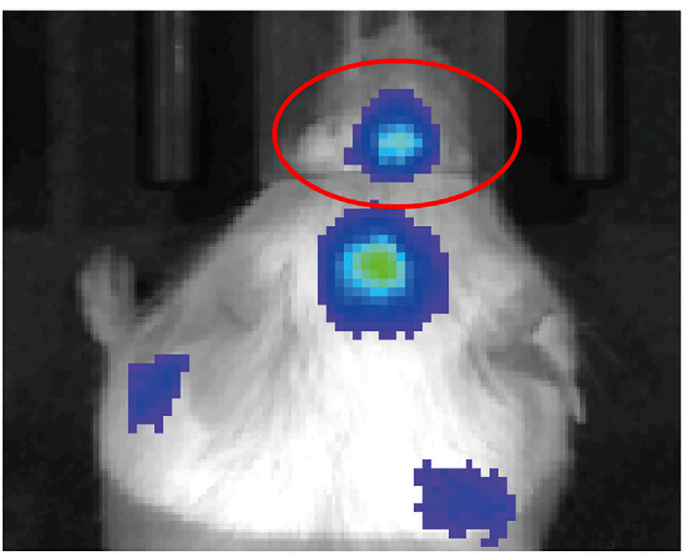

D-Luciferin

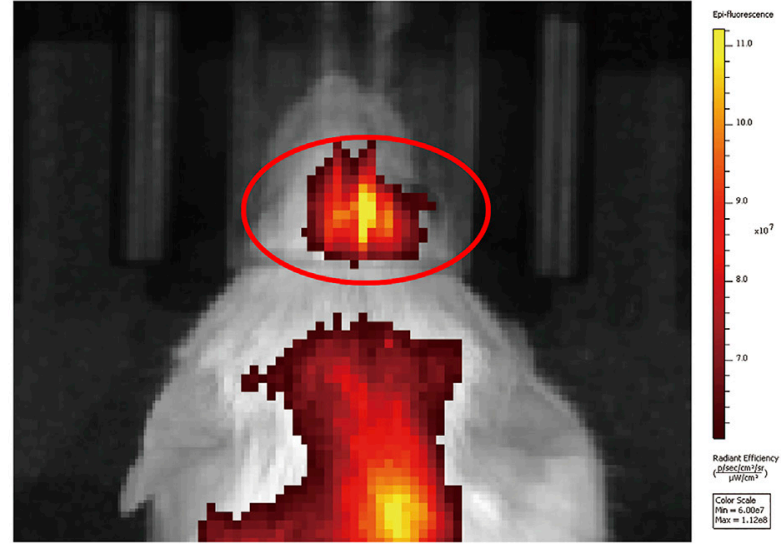

Cy5-S5
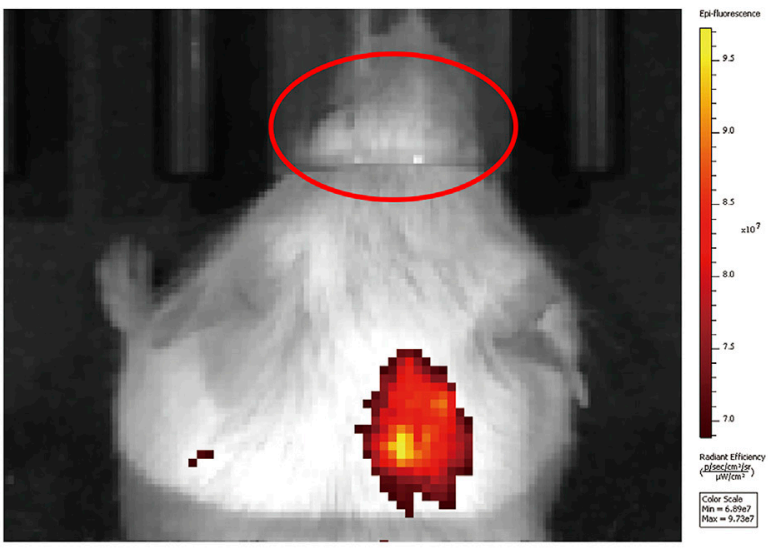

Control: Cy5-CG7C

FIGURE 2 | NIFR imaging with Cy5-S5 in ALL mouse models verified by bioluminescence imaging. (A) Cy5-S5 exhibited intensive fluorescence intensity in the similar tumor location of bioluminescence imaging, while (B) Cy5-CG7C did not show significant fluorescence intensity.

Fluorescence signals from the tumor were recorded before and $48 \mathrm{~h}$ after intravenous injection of the probes.

\section{MR Imaging}

Bioluminescence confirmed CNS-L mice were used for MR and PET imaging studies in the next few days after the NIRF imaging. MR imaging was performed using a Philips Achieva 3.0 T system. Mice were anesthetized with 2,2,2-Tribromoethanol $(100 \mu \mathrm{l} / \mathrm{g})$, fixed on the holder, and placed into the mouse-imaging coil. GdS5 or a control agent was delivered by the tail vein at a dose of $0.03 \mathrm{mmol}$ of $\mathrm{Gd} / \mathrm{kg}$ for the T1 mapping sequence in NALM6luciferase tumor-bearing mice. The clinical commonly used MR contrast medium gadolinium-diethylenetriamine pentaacetic acid (Gd-DTPA), which is a nonspecific agent, was used as control. On two separate days, mice received either a control enhance-agent $(0.03 \mathrm{mmol}$ of $\mathrm{Gd} / \mathrm{kg})$ or $\mathrm{Gd}-\mathrm{S} 5(0.03 \mathrm{mmol}$ of $\mathrm{Gd} / \mathrm{kg}$ ) injection and subsequent MR imaging, with at least 3 days between the scans to ensure most of the gadolinium were cleared. Axial T1-weighted images were acquired by using a gradient-echo sequence with the following parameters: $\mathrm{TR} / \mathrm{TE}=8.771 /$ $2.878 \mathrm{~ms}$, FOV $=4.0 \mathrm{~cm}$, slice thickness $=-1 \mathrm{~mm}$, slice spacing $=0.5$, pixel spacing $=0.234 \mathrm{~mm} \times 0.234 \mathrm{~mm}$, and matrix $=128 \times 128$.

\section{PET Imaging}

PET imaging was performed using a hybrid 3.0T PET/MR scanner (uPMR 790, United Imaging Healthcare). As described above, mice were anesthetized with 2,2,2Tribromoethanol $(100 \mu \mathrm{l} / \mathrm{g})$, fixed on the holder, and placed into the mouse-imaging coil. Approximately, $3.7 \times 10^{6} \mathrm{~Bq}$ $(100 \mu \mathrm{Ci})$ of ${ }^{18} \mathrm{~F}-\mathrm{S} 5$ or the clinical commonly used PET tracer ${ }^{18} \mathrm{~F}$-fluorodeoxyglucose (FDG) as control was injected via the tail vein before PET imaging. PET scans of the tumor-bearing mice were performed $60 \mathrm{~min}$ after 
A
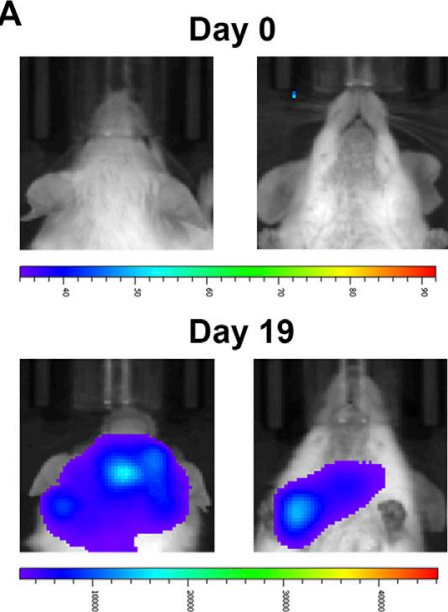

C

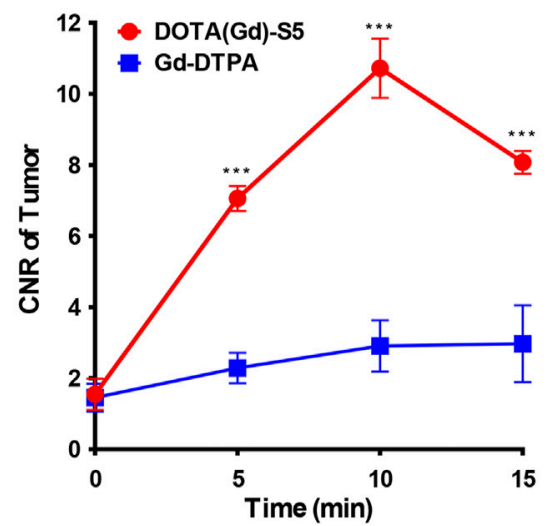

B
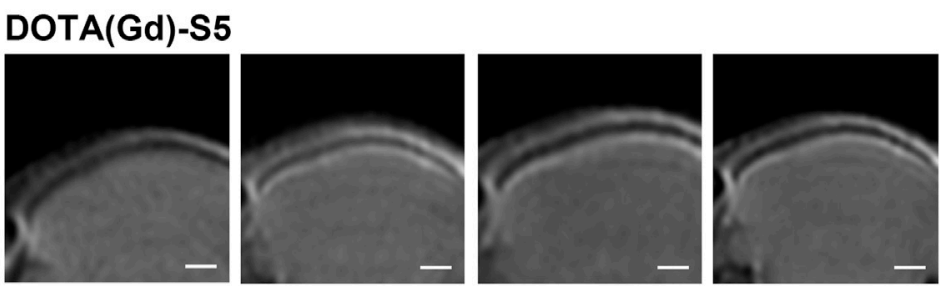

Flase color

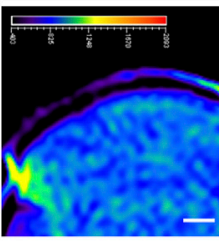

Pre

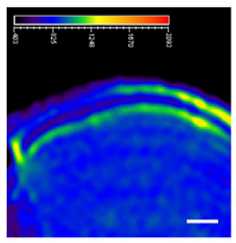

$5 \mathrm{~min}$
Control: Gd-DTPA
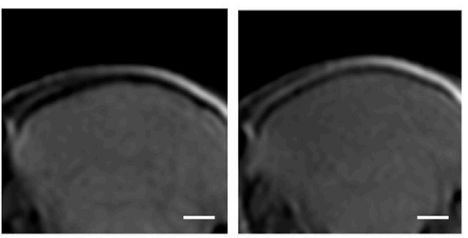

Flase color

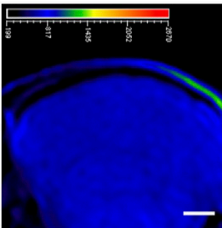

Pre

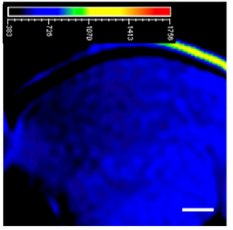

$5 \mathrm{~min}$

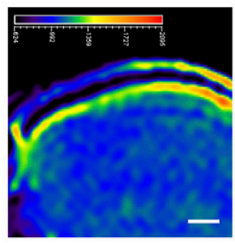

$10 \mathrm{~min}$
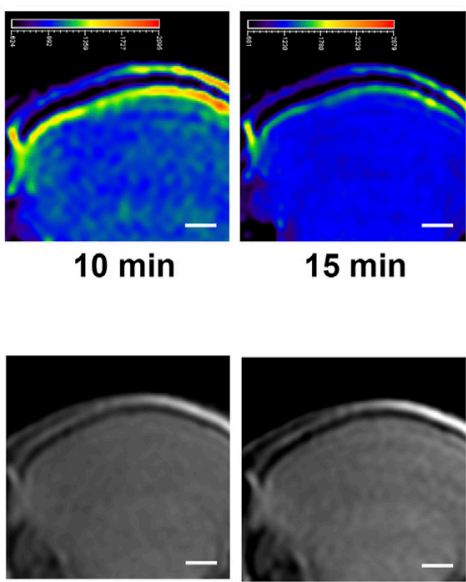

$15 \min$
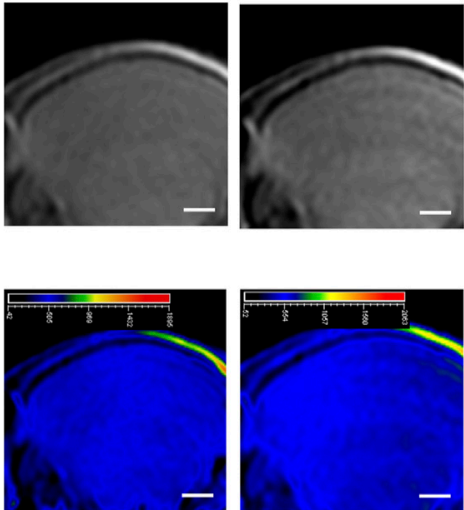

$10 \mathrm{~min}$

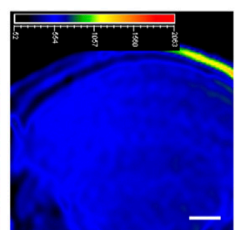

$15 \min$

FIGURE 3 | MR imaging with Gd-S5 in ALL mouse models. (A) Bioluminescence imaging exhibited focal luminescence intensity around the head 19 days after injection of NALM6-luciferased cells. (B) T1-weighted MR images at the baseline and 5, 10, and 15 min post-injection of Gd-S5 and the control probe Gd-DTPA (Scale bar, $1 \mathrm{~mm}$ ). (C) Gd-S5 generated a significantly higher signal enhancement at the site of meninges which were located between the skull bone and brain parenchyma at 5,10 , and 15 min post-injection, with the greatest gap at about 10 min post-injection $(n=3)$.

injection, with the following parameters: $\mathrm{TR} / \mathrm{TE}=8.771 /$ $2.878 \mathrm{~ms}, \mathrm{FOV}=4.0 \mathrm{~cm}$, slice thickness $=1 \mathrm{~mm}$, slice spacing $=0.5$, pixel spacing $=0.234 \mathrm{~mm} \times 0.234 \mathrm{~mm}$, and matrix $=$ $128 \times 128$.

\section{HE Staining and Immunohistochemistry}

At the end of imaging experiments, the mice were sacrificed by cervical dislocation and immediately dissected. The head part was collected, and haired skin and soft tissue were removed from the cranial surface. Paraffin sections $(3 \mu \mathrm{m})$ were stained with hematoxylin and eosin (H\&E) for routine histologic practice. Immunohistochemical (IHC) staining was performed following the conventional procedure, as we reported previously (Feng GK et al., 2019). Briefly, paraffin sections were dewaxed into xylene, rehydrated through graded alcohol, and microwaved for antigen retrieval. Blocking to inhibit the endogenous peroxidase activity and nonspecific binding, the sections were incubated with an anti-integrin a6 antibody (Abcam, ab181551, and 1:150) overnight at $4^{\circ} \mathrm{C}$, followed by an HRP-conjugated polyclonal secondary antibody $(1: 200)$ at room temperature for $1 \mathrm{~h}$. Finally, the positive immunoreactivity was visualized by staining with DAB (Zhongshan Jinqiao, ZLI-9017, China) and observed under a microscope (Nikon Eclipse, Japan).

\section{Statistical Analysis}

Statistical analysis was performed with SPSS 19.0 software (SPSS Inc., Chicago, United States). The significance was tested by the two-tailed Student's t-test, and a $p$ value less than 0.05 was considered to be statistically significant.

\section{RESULTS}

\section{Surface Expression of Integrin a6 in Human} ALL Cell Lines

The surface expression of integrin a6 was assayed by flow cytometry in human ALL cell lines including NALM6, Ref, Jurkat, and CCRF-CEM. As expected, flow cytometry 


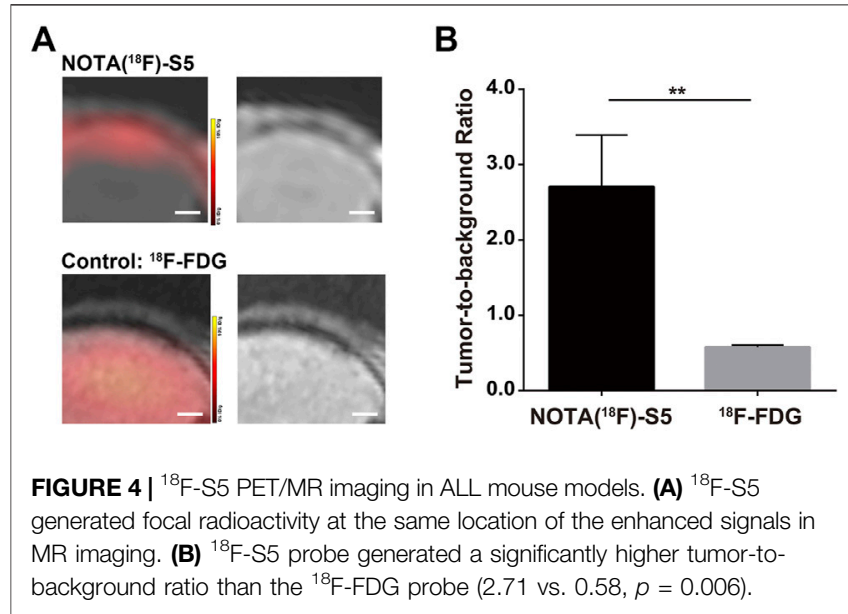

confirmed the surface expression of integrin $a 6$ in all the cell lines (Figure 1A). In Figure 1B, we describe the mean fluorescence intensity (MFI) of different cell flow assays. MFI means total fluorescence intensity divided by the number of positive cells. The highest expression of integrin a6 was observed in NALM6 cells with a positive rate of $99.8 \%$ (Figure 1B), which was used to establish ALL models for subsequent imaging experiments.

\section{Synthesis and Characterization}

The S5 peptide was synthesized using Fmoc-based solid-phase synthesis. Then, the S5 peptide was condensed with a PEG4 spacer and the Cy5, NOTA, or DOTA structure to furnish the Cy5-S5, NOTA-S5, and DOTA-S5. The purity of Cy5-S5, NOTA-S5, and DOTA-S5 was over 95\% by highperformance liquid chromatography (HPLC). The molecular weight was measured to be $1985,1,632$, and 1,435 by MALDITOF mass spectrometry, which was in agreement with the theoretical molecular weight calculated from the predicted amino acid sequence. Then, the DOTA-S5 was complexed with $\mathrm{Gd}$, and the NOTA-S5 peptide was radiolabeled with the radionuclide ${ }^{18} \mathrm{~F}$ to form the MR enhancer Gd-S5 and the PET radiotracer ${ }^{18} \mathrm{~F}$-S5 (Supplementary Figure S1). The structural formula used for the Gd-S5 peptide used for MR imaging is a straight peptide, based on the convenience and requirements of the synthesis process and the fact that our preliminary experiments have confirmed that the binding of cyclic and straight peptides is equivalent. The corresponding results are supplemented in Supplementary Figures S2A, S2B. In addition, the binding experiments with S5 carrying NOTA groups found that the attachment of NOTA did not affect the binding affinity of the peptide (Supplementary Figures S2C, S2D).

\section{Biding of the S5 Peptide to NALM6 Cells and NIRF Imaging}

The cellular localization of the S5 peptide in NALM6 cells was visualized by confocal microscopy. Green fluorescence represented the S5 polypeptide, which entered the cytoplasm through cytocytosis after binding to the cell
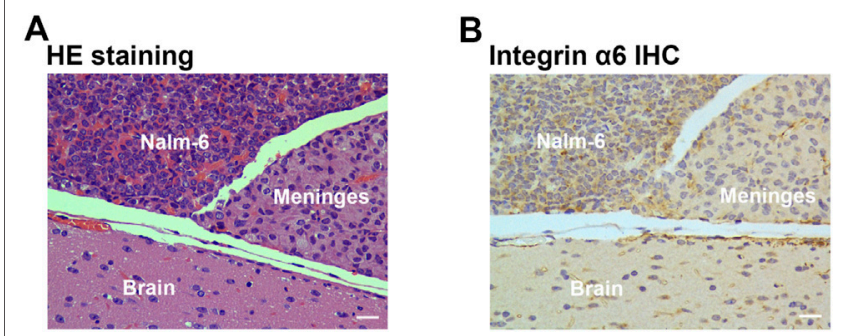

FIGURE 5 | Pathological examination of tumor tissue. HE staining (A) identified layer-displayed leukemic cells in the superficial part of the brain parenchyma tissue, and immunohistochemical staining (B) confirmed the integrin a6 overexpression of the lesion (Scale bar, $40 \mu \mathrm{m}$ ).

membrane. Red fluorescence represented the integrin a6 antibody, which was bound to the cell membrane and was then immobilized on the cell membrane. According to the fluorescence microscopy data, the S5 peptide was enriched and localized at the nuclei of NALM6 cells after $4 \mathrm{~h}$ of co-culturing (Figure 1C), indicating the targeted ability of the S5 peptide to integrin $a 6$ that was dominantly distributed on the nuclei. To further investigate the in vivo tumor-binding ability of the S5 peptide, NIRF imaging with Cy5-S5 and Cy5-CG7C (negative control) was conducted. The NALM6-luciferase tumorbearing mice were verified by bioluminescence imaging with a focal luminescence intensity around the head, and in NIRF imaging, Cy5-S5 exhibited intensive fluorescence intensity in the similar tumor location (Figure 2A), while the control imaging group with Cy5-CG7C did not show significant fluorescence intensity (Figure 2B).

\section{MR Imaging in NALM6-Luciferase Tumor-Bearing Mice}

Similarly, a leukemia CNS-L mouse model using NALM6 cells with the luciferase expression by tail vein injection was established. Approximately 3 weeks after tumor cell injection, the presence of focal luminescence intensity around the head was confirmed by bioluminescence imaging (Figure 3A). T1-weighted MR imaging was conducted before and at different time points after Gd-S5 or GdDTPA injection. To improve visibility, MR signals were also displayed in rainbow pseudo color. As shown in Figure 3B, Gd-S5 gradually produced contrast enhancement signals at the site of meninges located between the skull bone and brain parenchyma at $5 \mathrm{~min}$ post-injection, which conformed to the primary intracranial infiltrating location of CNS-L (Yao et al., 2018), reaching the peak at $10 \mathrm{~min}$, and gradually decreased thereafter. The control group with Gd-DTPA injection did not show obvious enhancement signals. The signal intensity comparison according to the post-injection duration is presented in Figure 3C, indicating that Gd-S5 generated significantly higher signals than Gd-DTPA. 


\section{PET Imaging in NALM6-Luciferase Tumor-Bearing Mice}

The PET imaging for ${ }^{18} \mathrm{~F}$-S5 was conducted $60 \mathrm{~min}$ after injection of the radiotracer through the tail vein. ${ }^{18} \mathrm{~F}-\mathrm{S} 5$ generated focal radioactivity at the site of meninges which is at the same location of enhanced signals generated by Gd-S5 in the previous MR imaging, whereas the brain parenchyma did not show significant radioactivity (Figure 4A). For the clinical commonly used PET radiotracer ${ }^{18} \mathrm{~F}$-FDG, it generated diffuse enhanced radioactivity in the brain tissue due to the hypermetabolism of glucose (Figure 4A). The tumor-to-background ratio of the ${ }^{18} \mathrm{~F}-\mathrm{S} 5$ probe was significantly higher than that of the ${ }^{18} \mathrm{~F}-\mathrm{FDG}$ probe (2.71 vs. $0.58, p=0.006$; Figure $4 B$ ).

\section{HE Staining and Immunohistochemistry}

At the end of the imaging experiments, mice were euthanized, and tumor tissues were removed for pathological examination. Pathological examination identified layer-displayed leukemic cells in the superficial part of the brain parenchyma tissue, and immunohistochemical staining confirmed the overexpression of integrin $a 6$ within the lesion (Figure 5).

\section{DISCUSSION}

To date, the detection of CNS-L still remains a great challenge due to the lack of accurate diagnostic tools. CNS-L could occur at any time during the full course of leukemia, when the disease is apparently well-controlled even during relapse. Current diagnostic approaches including CNS symptoms and pathological detection of leukemic cells in the cerebrospinal fluid are generally hysteretic than the primary CNS involvement. Imaging detection of CNS-L lesions could be more distinct. MRI provides excellent soft tissue contrast and spatial resolution without ionizing radiation, which has been widely used due to its relatively low cost and easy accessibility (Mirzaei and Adeli, 2018). Meanwhile, PET imaging has been widely utilized in the diagnosis and management of cancer patients with relatively high sensitivity and quantitativeimaging ability (Shankar et al., 2020; Overcast et al., 2021). However, these imaging examinations have showed a limited ability in the imaging of CNS-L mainly due to the special histopathological characteristics of the disease.

Meningeal infiltration through the penetration of sinuses and small blood vessels is the earliest pathological process at the occurrence of CNS-L, followed by parenchymal infiltration, leukostasis, meningitis, intracranial edema, hemorrhage, and herniation (Yao et al., 2018; Halsey and Escherich, 2021). Distinctive from the other intracranial tumors, solid lesions hardly formed during the course of CNS-L, which caused a great challenge for conventional imaging methods (Pfeifer et al., 2003; Izraeli and Eckert, 2017). In addition, because of the physiological glucose hypermetabolism of a normal brain tissue, the most commonly used PET tracer ${ }^{18}$ F-FDG was less applicable to the intracranial lesions. Based on the premises, the development of novel molecular imaging probes with targeted ability could offer novel opportunities for the imaging of CNS-L.

Here, we focused on the potential tumor biomarker integrin a6, which was found to be overexpressed in a series of cancers (Huang et al., 2012; Brooks et al., 2016; Beaulieu, 2018). Physiologically, integrin a6 was mainly involved in cell proliferation, migration, survival, and differentiation by mediating cell-to-cell and cell-to-stroma adhesion. The expression and function of integrin a6 were abnormally altered in tumor cells, which promoted tumorigenesis, invasion, angiogenesis, metastasis, immune escape, and tolerance to radiotherapy and chemotherapy (Lathia et al., 2010; Beaulieu, 2019). In ALL, integrin $\alpha 6$ has been implicated to be crucial in the migration into CNS tissues and promote the survival of minimal residual disease after chemotherapy (Yao et al., 2018). Besides, silencing of integrin a 6 could induce apoptosis and sensitize ALL cells to nilotinib or chemotherapy regimens, suggesting its important role in mediating chemoresistance (Yamakawa et al., 2012; Gang et al., 2020). As a membrane protein, integrin $a 6$ also had a relatively long $\mathrm{N}$-terminal extracellular domain that could be approached and bounded by targeted agents and which rendered it a potentially suitable target for molecular imaging and antitumor therapy.

In this study, the overexpression of integrin a 6 was identified in numerous ALL cell lines including NALM6, REH, and CCRF-CEM cells, and the dominating membrane location of integrin $a 6$ and the S5 peptide was confirmed by confocal microscopy. Then, the S5 peptide-based NIRF-, MRI-, and PET-targeted imaging probes were successfully constructed in four major domains: the S5 peptide domain for tumor targeting; Cy5, Gd, or ${ }^{18} \mathrm{~F}$ for the NIRF tracer, MRI contrast enhancement, or PET radiotracer; DOTA or NOTA as a chemical complexation ligand for linking $\mathrm{Gd}$ or ${ }^{18} \mathrm{~F}$; and a PEG4 spacer to avoid the steric hindrance of the Cy5, GdDOTA, or ${ }^{18}$ F-NOTA monoamide to the S5 peptide. The synthesized agents Cy5-S5, Gd-S5, and ${ }^{18} \mathrm{~F}-\mathrm{S} 5$ were hydrophilic and had good water solubility, suggesting its potential for further imaging development.

Our results illustrated that the gradually enhanced MRI signals were acquired at the site of meninges that were located between the skull bone and brain parenchyma of the mouse models, which was in accordance with the primary intracranial infiltrating location of CNS-L (Yao et al., 2018), reaching the peak at around $10 \mathrm{~min}$, which was significantly highlighted compared to the surrounded brain tissues and easy to be recognized in visual, whereas the MRI images of the control group did not show significant enhancement signals. The quantitative signal analysis revealed that the S5 peptide-based probe generated, in approximately, signal enhancement 3 folds more than the control agent within the first $5 \mathrm{~min}$ post-injection, and the gap widened to nearly 4 folds at 10 min after injection. The signal enhancement gradually decreased thereafter and was still distinctive at 15 min post-injection. The PET imaging also showed regional radiation concentration at the same site of the MRI signal enhancement. Although the absolute SUV 
value was not high, its relative ratio to the brain parenchymal tissue reached near 3 folds, indicating considerable imaging ability of the S5-based PET probe.

Tumor-specific antibodies have been developed for targeted imaging; however, typically several days were needed to achieve the optimal imaging condition due to the long blood circulation duration of the antibodies. Targeted peptides have several advantages compared to the antibodies including the small size, sufficient capillary permeability, low immunogenicity, short biological half-time, rapid clearance from non-target tissues, ease of manufacture, and readily labeled with specific nuclides (Lee et al., 2010; Yao et al., 2016; Araste et al., 2018). In our study, Gd-S5 and ${ }^{18} \mathrm{~F}$-S5 could be quickly excreted through the kidney due to its small molecular structure and good permeability, which enables the basis for imaging safety.

On the other hand, our study also has several limitations. First, the diagnostic efficacy of the molecular probes, compared to conventional cerebrospinal fluid examinations that are routinely conducted in clinics, was not investigated and could be further identified in larger animal models in which the cerebrospinal fluid is more accessible. Besides, the binding affinity of the S5 peptide to integrin a6 could be further enhanced through optimizing the peptide structure.

In conclusion, the study demonstrated that integrin a 6 could serve as a feasible target for the molecular targeted imaging of CNS-L. The S5 peptide-based MRI and PET enhancement probes showed considerable ability in the imaging of CNS-L lesions in mouse models, indicating their potential for further development and clinical translation.

\section{DATA AVAILABILITY STATEMENT}

The original contributions presented in the study are included in the article/Supplementary Material, further inquiries can be directed to the corresponding authors. The authenticity of this article has been validated by uploading the key raw data onto the

\section{REFERENCES}

Araste, F., Abnous, K., Hashemi, M., Taghdisi, S. M., Ramezani, M., and Alibolandi, M. (2018). Peptide-based Targeted Therapeutics: Focus on Cancer Treatment. J. Control. Release 292, 141-162. doi:10.1016/j.jconrel. 2018.11.004

Beaulieu, J.-F. (2018). Integrin $\hat{\mathrm{I}} \pm 6$ Variants and Colorectal Cancer. Gut 67, 1747-1748. doi:10.1136/gutjnl-2017-315415

Beaulieu, J.-F. (2019). Integrin $\alpha 6 \beta 4$ in Colorectal Cancer: Expression, Regulation, Functional Alterations and Use as a Biomarker. Cancers 12, 41. doi:10.3390/ cancers 12010041

Blase, L., Merling, A., Engelmann, S., Möller, P., and Schwartz-Albiez, R. (1996). Characterization of Cell Surface-Expressed Proteochondroitin Sulfate of Pre-B Nalm-6 Cells and its Possible Role in Laminin Adhesion. Leukemia 10, 1000-1011.

Brooks, D. L. P., Schwab, L. P., Krutilina, R., Parke, D. N., Sethuraman, A., Hoogewijs, D., et al. (2016). ITGA6 Is Directly Regulated by HypoxiaInducible Factors and Enriches for Cancer Stem Cell Activity and Invasion in Metastatic Breast Cancer Models. Mol. Cancer 15, 26. doi:10.1186/s12943-016-0510-x
Research Data Deposit public platform (www.researchdata.org. cn), with the approval RDD number as RDDB2022504768.

\section{ETHICS STATEMENT}

The animal study was reviewed and approved by the Ethics Committee of the Sun Yat-sen University Cancer Center.

\section{AUTHOR CONTRIBUTIONS}

HW, GF, and XZ conceived the presented idea. GF developed the linkage mode of the tracer. $\mathrm{XZ}$ developed the chemical structure of tracers and the radiosynthesis method. HW designed the imaging verifying methods. WZ, YL, and GC conducted the experiments. WZ carried out the cell line constructions and in vitro studies. YL performed the imaging scans. GC carried out the tracer labeling and administration. $\mathrm{XC}$ and $\mathrm{JH}$ provided the study materials and technique support. Rodents used in the experiments were provided by XC. Confocal images and PET images were obtained with the help of $\mathrm{JH}$.

\section{FUNDING}

This work was carried out with the support of the National Natural Science Foundation of China (NSFC) (Grant: 81700148) and the Natural Science Foundation of Guangdong Province Funding (Grant: 2021A1515010093).

\section{SUPPLEMENTARY MATERIAL}

The Supplementary Material for this article can be found online at: https://www.frontiersin.org/articles/10.3389/fbioe. 2022.812277/full\#supplementary-material

Bürger, B., Zimmermann, M., Mann, G., Kühl, J., Löning, L., Riehm, H., et al. (2003). Diagnostic Cerebrospinal Fluid Examination in Children with Acute Lymphoblastic Leukemia: Significance of Low Leukocyte Counts with Blasts or Traumatic Lumbar Puncture. J. Clin. Oncol. 21, 184-188. doi:10.1200/jco.2003. 04.096

Erdreich-Epstein, A., Tran, L. B., Cox, O. T., Huang, E. Y., Laug, W. E., Shimada, H., et al. (2005). Endothelial Apoptosis Induced by Inhibition of Integrins $\alpha v \beta 3$ and av $\beta 5$ Involves Ceramide Metabolic Pathways. Blood 105, 4353-4361. doi:10.1182/blood-2004-08-3098

Feng G, G., Zhang, M., Wang, H., Cai, J., Chen, S., Wang, Q., et al. (2019). Identification of an Integrin a6-Targeted Peptide for Nasopharyngeal Carcinoma-specific Nanotherapeutics. Adv. Ther. 2, 1900018. doi:10.1002/ adtp. 201900018

Feng GK, G.-K., Ye, J.-C., Zhang, W.-G., Mei, Y., Zhou, C., Xiao, Y.-T., et al. (2019). Integrin a6 Targeted Positron Emission Tomography Imaging of Hepatocellular Carcinoma in Mouse Models. J. Control. Release 310, 11-21. doi:10.1016/j.jconrel.2019.08.003

Frishman-Levy, L., and Izraeli, S. (2017). Advances in Understanding the Pathogenesis of CNS Acute Lymphoblastic Leukaemia and Potential for Therapy. Br. J. Haematol. 176, 157-167. doi:10.1111/bjh.14411 
Gang, E. J., Kim, H. N., Hsieh, Y.-T., Ruan, Y., Ogana, H. A., Lee, S., et al. (2020). Integrin $\alpha 6$ Mediates the Drug Resistance of Acute Lymphoblastic B-Cell Leukemia. Blood 136, 210-223. doi:10.1182/blood.2019001417

Gao, S., Jia, B., Feng, G., Dong, C., Du, H., Bai, L., et al. (2020). First-inhuman Pilot Study of an Integrin a6-targeted Radiotracer for SPECT Imaging of Breast Cancer. Sig. Transduct. Target. Ther. 5, 147. doi:10. 1038/s41392-020-00266-9

Halsey, C., and Escherich, G. (2021). A "Goldilocks" Approach to CNS Leukemia Is Needed. Blood 138, 288-289. doi:10.1182/blood.2021011461

Huang, P., Rani, M. R. S., Ahluwalia, M. S., Bae, E., Prayson, R. A., Weil, R. J., et al. (2012). Endothelial Expression of TNF Receptor-1 Generates a Proapoptotic Signal Inhibited by Integrin $\alpha 6 \beta 1$ in Glioblastoma. Cancer Res. 72, 1428-1437. doi:10.1158/0008-5472.can-11-2621

Izraeli, S., and Eckert, C. (2017). Targeted Therapy of CNS Leukemia? Blood 130, 562-563. doi:10.1182/blood-2017-06-788430

Jin, M. W., Xu, S. M., and An, Q. (2018). Central Nervous Disease in Pediatric Patients during Acute Lymphoblastic Leukemia (ALL): a Review. Eur. Rev. Med. Pharmacol. Sci. 22, 6015-6019. doi:10.26355/ eurrev_201809_15937

Krebsbach, P. H., and Villa-Diaz, L. G. (2017). The Role of Integrin a6 (CD49f) in Stem Cells: More Than a Conserved Biomarker. Stem Cell Dev. 26, 1090-1099. doi:10.1089/scd.2016.0319

Lathia, J. D., Gallagher, J., Heddleston, J. M., Wang, J., Eyler, C. E., Macswords, J., et al. (2010). Integrin Alpha 6 Regulates Glioblastoma Stem Cells. Cell Stem Cell 6, 421-432. doi:10.1016/j.stem.2010.02.018

Lee, S., Xie, J., and Chen, X. (2010). Peptides and Peptide Hormones for Molecular Imaging and Disease Diagnosis. Chem. Rev. 110, 3087-3111. doi:10.1021/ cr900361p

Lenk, L., Alsadeq, A., and Schewe, D. M. (2020). Involvement of the central Nervous System in Acute Lymphoblastic Leukemia: Opinions on Molecular Mechanisms and Clinical Implications Based on Recent Data. Cancer Metastas. Rev. 39, 173-187. doi:10.1007/s10555-020-09848-Z

Lin, B.-Q., Zhang, W.-B., Zhao, J., Zhou, X.-H., Li, Y.-J., Deng, J., et al. (2021). An Optimized Integrin a6-Targeted Magnetic Resonance Probe for Molecular Imaging of Hepatocellular Carcinoma in Mice. J. Hepatocell. Carcinoma 8, 645-656. doi:10.2147/jhc.s312921

Malard, F., and Mohty, M. (2020). Acute Lymphoblastic Leukaemia. Lancet 395, 1146-1162. doi:10.1016/s0140-6736(19)33018-1

Mei, Y., Li, Y. H., Yang, X. C., Zhou, C., Li, Z. J., Zheng, X. B., et al. (2020). An Optimized Integrin a6-targeted Peptide for Positron Emission Tomography/ magnetic Resonance Imaging of Pancreatic Cancer and its Precancerous Lesion. Clin. Transl. Med. 10, e157. doi:10.1002/ctm2.157

Mirzaei, G., and Adeli, H. (2018). Segmentation and Clustering in Brain MRI Imaging. Rev. Neurosci. 30, 31-44. doi:10.1515/revneuro-2018-0050

Overcast, W. B., Davis, K. M., Ho, C. Y., Hutchins, G. D., Green, M. A., Graner, B. D., et al. (2021). Advanced Imaging Techniques for NeuroOncologic Tumor Diagnosis, with an Emphasis on PET-MRI Imaging of Malignant Brain Tumors. Curr. Oncol. Rep. 23, 34. doi:10.1007/s11912021-01020-2

Pfeifer, H., Wassmann, B., Hofmann, W. K., Komor, M., Scheuring, U., Brück, P., et al. (2003). Risk and Prognosis of central Nervous System Leukemia in Patients with Philadelphia Chromosome-Positive Acute Leukemias Treated with Imatinib Mesylate. Clin. Cancer Res. 9, 4674-4681.

Pinnix, C. C., Yahalom, J., Specht, L., and Dabaja, B. S. (2018). Radiation in Central Nervous System Leukemia: Guidelines from the International Lymphoma Radiation Oncology Group. Int. J. Radiat. Oncol. Biol. Phys. 102, 53-58. doi:10.1016/j.ijrobp.2018.05.067

Ranta, S., Palomäki, M., Levinsen, M., Taskinen, M., Abrahamsson, J., Mellgren, K., et al. (2017). Role of Neuroimaging in Children with Acute Lymphoblastic Leukemia and central Nervous System Involvement at Diagnosis. Pediatr. Blood Cancer 64, 64-70. doi:10.1002/pbc.26182

Ranta, S., Palomäki, M., Levinsen, M., Taskinen, M., Abrahamsson, J., Hasle, H., et al. (2017). Presenting Features and Imaging in Childhood Acute Myeloid Leukemia with central Nervous System Involvement. Pediatr. Blood Cancer 64, e26459. doi:10.1002/pbc.26459
Shankar, A., Bomanji, J., and Hyare, H. (2020). Hybrid PET-MRI Imaging in Paediatric and TYA Brain Tumours: Clinical Applications and Challenges. J. Pers. Med. 10, 218. doi:10.3390/jpm10040218

Siegel, R. L., Miller, K. D., and Jemal, A. (2019). Cancer Statistics, 2019. CA Cancer J. Clin. 69, 7-34. doi:10.3322/caac.21551

Takada, Y., Ye, X., and Simon, S. (2007). The Integrins. Genome Biol. 8, 215. doi:10. 1186/gb-2007-8-5-215

Thastrup, M., Marquart, H. V., Levinsen, M., Grell, K., Abrahamsson, J., Albertsen, B. K., et al. (2020). Flow Cytometric Detection of Leukemic Blasts in Cerebrospinal Fluid Predicts Risk of Relapse in Childhood Acute Lymphoblastic Leukemia: a Nordic Society of Pediatric Hematology and Oncology Study. Leukemia 34, 336-346. doi:10.1038/s41375-019-0570-1

Vagace, J. M., de la Maya, M. D., Caceres-Marzal, C., Gonzalez de Murillo, S., and Gervasini, G. (2012). Central Nervous System Chemotoxicity during Treatment of Pediatric Acute Lymphoblastic Leukemia/lymphoma. Crit. Rev. Oncol. Hematol. 84, 274-286. doi:10.1016/j.critrevonc.2012.04.003

Xiao, Y.-T., Zhou, C., Ye, J.-C., Yang, X.-C., Li, Z.-J., Zheng, X.-B., et al. (2019). Integrin a6-Targeted Positron Emission Tomography Imaging of Colorectal Cancer. ACS Omega 4, 15560-15566. doi:10.1021/acsomega.9b01920

Yamakawa, N., Kaneda, K., Saito, Y., Ichihara, E., and Morishita, K. (2012). The Increased Expression of Integrin a6 (ITGA6) Enhances Drug Resistance in EVI1high Leukemia. PloS one 7, e30706. doi:10.1371/journal.pone.0030706

Yao, V. J., D’Angelo, S., Butler, K. S., Theron, C., Smith, T. L., Marchiò, S., et al. (2016). Ligand-targeted Theranostic Nanomedicines against Cancer. J. Control. Release 240, 267-286. doi:10.1016/j.jconrel.2016.01.002

Yao, H., Price, T. T., Cantelli, G., Ngo, B., Warner, M. J., Olivere, L., et al. (2018). Leukaemia Hijacks a Neural Mechanism to Invade the central Nervous System. Nature 560, 55-60. doi:10.1038/s41586-018-0342-5

Yousafzai, Y. M., Smith, L., Smith, A., Bhatti, S., Gardiner, M., Cousins, A., et al. (2019). Use of Quantitative Polymerase Chain Reaction (qPCR) for the Diagnosis and Monitoring of CNS Leukaemia. Leuk. Res. 87, 106232. doi:10. 1016/j.leukres.2019.106232

Zhang C, C., Zhong, J. F., and Zhang, X. (2020). Revealing the Molecular Mechanism of central Nervous System Leukemia with Single-Cell Technology. Crit. Rev. Oncol. Hematol. 153, 103046. doi:10.1016/j. critrevonc.2020.103046

Zhang Y, Y., Zhao, J., Cai, J., Ye, J.-C., Xiao, Y.-T., Mei, Y., et al. (2020). Integrin a6Targeted Magnetic Resonance Imaging of Hepatocellular Carcinoma in Mice. Mol. Imaging Biol. 22, 864-872. doi:10.1007/s11307-019-01437-z

Zhou, C., Zhuang, Y., Lin, X., Michelson, A. D., and Zhang, A. (2020). Changes in Neurocognitive Function and central Nervous System Structure in Childhood Acute Lymphoblastic Leukaemia Survivors after Treatment: a Meta-analysis. Br. J. Haematol. 188, 945-961. doi:10.1111/bjh.16279

Zhu, J., Carman, C. V., Kim, M., Shimaoka, M., Springer, T. A., and Luo, B.-H. (2007). Requirement of $\alpha$ and $\beta$ Subunit Transmembrane helix Separation for Integrin Outside-In Signaling. Blood 110, 2475-2483. doi:10.1182/blood-200703-080077

Conflict of Interest: The authors declare that the research was conducted in the absence of any commercial or financial relationships that could be construed as a potential conflict of interest.

Publisher's Note: All claims expressed in this article are solely those of the authors and do not necessarily represent those of their affiliated organizations, or those of the publisher, the editors, and the reviewers. Any product that may be evaluated in this article, or claim that may be made by its manufacturer, is not guaranteed or endorsed by the publisher.

Copyright $\odot 2022$ Zhang, Li, Chen, Yang, Hu, Zhang, Feng and Wang. This is an open-access article distributed under the terms of the Creative Commons Attribution License (CC BY). The use, distribution or reproduction in other forums is permitted, provided the original author(s) and the copyright owner(s) are credited and that the original publication in this journal is cited, in accordance with accepted academic practice. No use, distribution or reproduction is permitted which does not comply with these terms. 